\title{
Drought assessment in the Pampanga River basin, the Philippines - Part 2: A comparative SPI approach for quantifying climate change hazards
}

\author{
$\underline{\text { A. Hasegawa }}^{\text {a }}$, M. Gusyev ${ }^{\mathrm{a}}$, T. Ushiyama ${ }^{\mathrm{a}}$, J. Magome ${ }^{\mathrm{b}}$ and Y. Iwami $^{\mathrm{a}}$ \\ a International Centre for Water Hazard and Risk Management (ICHARM), Public Works Research Institute, \\ Minamihara 1-6, Tsukuba, Ibaraki, Japan
${ }^{\mathrm{b}}$ International Research Center for River Basin Environment (ICRE), University of Yamanashi, \\ Takeda 4-3-11, Kofu, Yamanashi, Japan \\ Email: hase55@pwri.go.jp
}

\begin{abstract}
The change of climatic patterns is a major concern in the Pampanga River basin, the Philippines, and may intensify the magnitude of drought and flood hazard events under climate change uncertainty. Most of municipal water demand requirements for the Metro Manila relies on the Angat dam, which drains only $546 \mathrm{~km}^{2}$ at the headwaters of the Pampanga River basin, as mentioned in Part 1 of this study. In this study, the climate change impacts were investigated for the RCP8.5 greenhouse gas emission scenario using 13 of higher-resolution general circulation models (GCMs) in CMIP5 and 4 ensemble members of the 20-km super high resolution atmospheric GCM, MRI-AGCM3.2S, with different sea surface temperature (SST) and sea ice distributions. The simulated daily precipitation was bias-corrected with the daily gridded rain gauges dataset as a reference observation, after the bilinear interpolation from the coarse grids of GCMs to the fine grid of the observation dataset. To estimate the meteorological hazards of droughts and floods due to climate change, we compared the precipitation characteristics between present (1979-2003) and future (2075-2099) climate projections using the standardized precipitation index (SPI) and the comparative SPI (cSPI).
\end{abstract}

The cSPI is a new concept designed to intuitively evaluate the meteorological drought in target datasets on the basis of the reference dataset. Computing the SPI by substituting future climate precipitation in the cumulative distribution function (CDF) with parameters derived from the corresponding present climate, we can compute the cSPI in future climate projections on the basis of the present climate projection. The cSPI could estimate not only the probability change of extremes but also the change of mean precipitation due to climate change simultaneously.

From cSPI results, the interannual variability of the cSPI in future climates increased significantly, but the average of the cSPI was almost the same as in present climate projections in the SST ensemble average of MRI-AGCM3.2S. As the result, both the meteorological drought and flood increased in MRI-AGCM3.2S simulations. In the higher-resolution model ensemble average CMIP5, the interannual variability of the cSPI in future climate was almost the same as in present climate projections, but the average of the cSPI significantly increased. As the result, the meteorological drought might be decreased in future but the heavy wet conditions could be increased in CMIP5 multi-model ensembles. It would be required to adapt to the heavier wet condition in the Angat dam in the end of the 21st century under the RCP8.5 emission scenario than the present climate condition.

The comparative standardized index concept of the cSPI approach, computing standardized index by substituting target datasets in the $\mathrm{CDF}$ with parameters derived from the reference dataset, is applicable to the other standardized indices, such as the standardized streamflow index (SSI), the standardized reservoir storage index (SRSI), and the standardized precipitation evapotranspiration index (SPEI), which is obtained with various probability distributions. For example, the comparative SRSI is utilized to evaluate socio-economic droughts under climate change in Part 3 of this study.

Keywords: Standardized precipitation index (SPI), comparative SPI (cSPI), meteorological drought, precipitation, climate change 
Hasegawa et al., Drought assessment in the Pampanga River basin, the Philippines-Part 2: A comparative SPI...

\section{INTRODUCTION}

The standardized precipitation index (SPI) is a versatile tool to monitor and analyse the meteorological drought on multiple timescales (e.g., McKee et al., 1993; Edwards and McKee, 1997). The SPI is a powerful and flexible index with a simple calculation procedure. In the SPI calculation, the precipitation is the only required meteorological variable and is used as an input to compute cumulative distribution function (CDF) for the SPI basis. In 2009, the Lincoln Declaration on Drought Indices recommended that the use of SPI by national meteorological and hydrological services world-wide for characterizing the meteorological drought, in addition to other drought indices that were in use in their service (WMO, 2012).

Up to date, the SPI has been applied to the climate change analysis, not only for separated present and future climate models' runs, but also for a continuous precipitation time series of historical and future climate simulations, although temperature was not an input parameter in the SPI (e.g., Grillakis et al., 2011; Wang et al., 2011). In most cases, the SPI values were computed separately in present and future climates on each CDF basis or from the continuous precipitation between present to future climates. The SPI in future period has not been computed on the basis of present period CDF in both cases. Therefore, it was difficult to interpret the meteorological drought from the SPI without the present climate basis. In addition, this problem was also addressed in the other standardized indices, even if they were suitable to climate change issues.

This study proposes a comparative SPI (cSPI) approach to overcome this problem. Using the cSPI, we assessed the meteorological drought in future climate on the basis of present climate in the Angat dam catchment in the Pampanga River basin, the Philippines. Most of municipal water demand requirements for the Metro Manila is supplied from the Angat dam, which has a total water storage capacity of $988 \times 10^{6} \mathrm{~m}^{3}$ and an upstream catchment area of about $546 \mathrm{~km}^{2}$. Refer to Gusyev et al. (2015a) in more details of the basin.

\section{DATA AND METHODOLOGY}

This study was based on general circulation model (GCM) data separated into two groups of present and future climate projections. One was the time slice simulations under different boundary and initial conditions using MRI-AGCM3.2S with 20-km grid (Mizuta et al., 2012). An AMIP-type run using the observed boundary conditions was treated as a present climate, labeled as "p1". For future climates under the RCP8.5 scenario, Mizuta et al. (2014) prepared 4 different sea surface temperature (SST) distributions. The future climate projections with the distributions of the cluster 1, 2, 3, and total, described in Mizuta et al. (2014), were labeled as "f1" to "f4", respectively. The other was the historical and RCP8.5 experiments using 13 of the higher-resolution coupled GCMs (CGCMs) in CMIP5 (Taylor et al., 2012). The CGCMs as ACCESS1.0, ACCESS1.3, CCSM4, CESM1(BGC), CMCC-CMS, CMCC-CM, CNRM-CM5, CSIRO-Mk3.6.0, MIROC5, MPI-ESM-MR, MPI-ESM-LR, MRI-CGCM3, and BCC-CSM1.1(m), were labeled as "m1" to "m13". Based on the 25 years of MRI-AGCM3.2S runs, the present period was 1979 to 2003, and the future one was 2075 to 2099 in this study.

The gridded daily rain gauges dataset in Asia, APHRODITE V1101 (Yatagai et al., 2012), was used as a reference observation. All of the projected daily precipitation datasets were bias-corrected using a non-parametric method divided into 12 months and extremes (Inomata et al., 2011) after the bilinear interpolation from the GCMs to the observation grid system. Then, the bias-corrected daily precipitation was aggregated into monthly totals at each grid, and averaged over the Angat dam catchment in the Pampanga River basin. The monthly precipitation in $m$-th month $(1 \leq m \leq 12)$ in a given year was aggregated at $S$-month timescale of the SPI (e.g., usually 1-, 3-, 6-, 9- and 12-month).

From the precipitation data, we derive the CDF of the Gamma distribution and the corresponding shape and scale parameters, $\alpha$ and $\beta$, with the maximum likelihood method as:

$$
G(x)=\frac{1}{\beta^{\alpha} \Gamma(\alpha)} \int_{0}^{x} x^{\alpha-1} e^{-x / \beta} d x, \text { where } \alpha=\frac{1}{4 A}\left(1+\sqrt{1+\frac{4 A}{3}}\right) \quad \text { and } \beta=\frac{\bar{x}}{\alpha}
$$

where $\Gamma(\alpha)$ is the ordinary gamma function of $\alpha, A=\ln \bar{x}-\frac{\sum \ln x}{n}, \bar{x}=\frac{\sum x}{n}$, and $n$ is the number of the values of $x$ in the period. Substituting the aggregated precipitation for $x$, we can estimate a pair of the Gamma distribution parameters and the CDF in equation (1) of a given $m$-th month at $S$-month timescale. The CDF is transformed into a standardized normal distribution as the SPI of a given $m$-th month at $S$-month timescale, if the precipitation dataset does not contain any zero values. The above procedures are described in the previous studies (e.g., Edwards and McKee, 1997). 
The precipitation dataset may practically contain zero values, even if the Gamma distribution is undefined for zero precipitation. To avoid this problem, many of the SPI studies modified the CDF as:

$$
H(x)=q+(1-q) G(x),
$$

where $q$ is the probability of zero precipitation, which can be handled in two ways as introduced by Loukas and Vasiliades (2004). In this study, we substituted the zero precipitation with a small amount of precipitation, such as $1 \mathrm{~mm}$, mentioned as quite "naïve" procedure in Loukas and Vasiliades (2004).

\section{SPI CLIMATE CHANGE ASSESSMENT}

As an example of present climate runs, Figure 1a shows the time series of $S P I 12_{\mathrm{p}}$ at 12 -month timescale of $\mathrm{p} 1$ run of MRI-AGCM3.2S. The subscripts "p" and "f" mean present and future climate projections, hereafter. The SPI value means drier in the negative case, and wetter in the positive case on the basis of the climate condition. As the absolute values of SPI is increased, the degrees of dryness and wetness are increased such as "moderately" $(1 \leq|S P I|<1.5)$, "severely" $(1.5 \leq|S P I|<2)$, and "extremely" $(|S P I| \geq 2)$. The SPI in $[-1,1]$ means "normal" condition of the climate. The moderately and severely zones of dryness and wetness were shaded with light (yellow and sky blue) and dark (orange and cyan) colors in the figures, respectively. Note that the timing of the dryness and wetness in the present climate simulations does not conform to that in the observation, even if the bias-

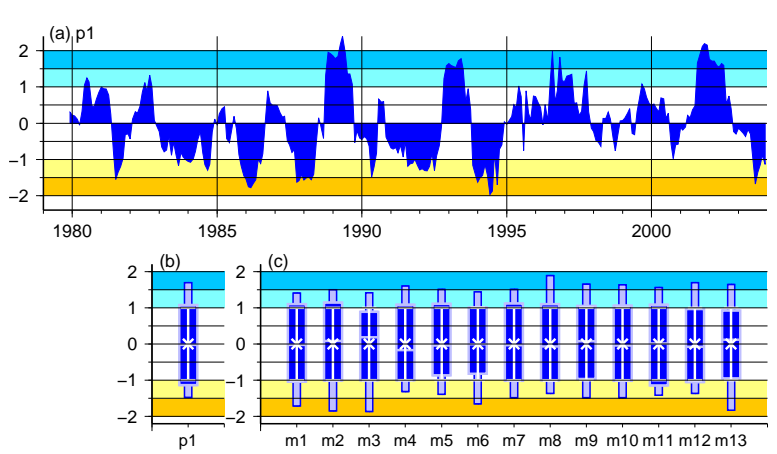

Figure 1. Time series of $S P I 12_{\mathrm{p}}$ of $\mathrm{p} 1$ run of MRIAGCM3.2S (a), and variability ranges of $S P I 12_{\mathrm{p}}$ of present climate rus using (b) MRI-AGCM3.2S and (c) 13 CGCMs in CMIP5. The cross and middle bar of wide box show the mean and median for each run. corrected precipitation of AMIP-type runs with the super-high-resolution GCMs, because of the climate projections without any data assimilations.

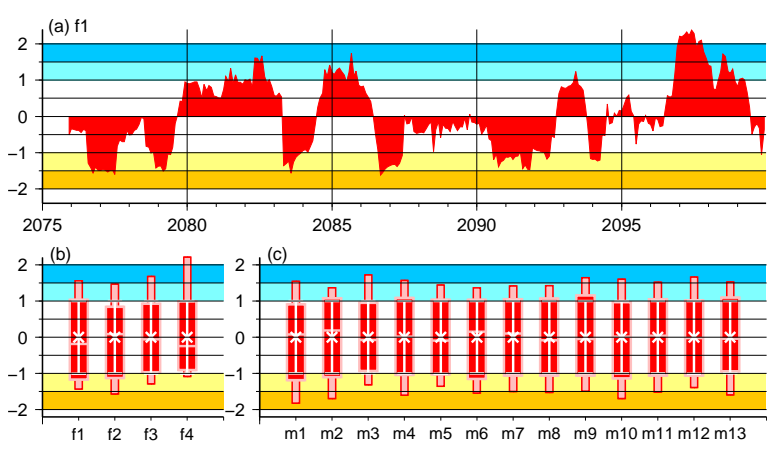

Figure 2. Same as Figure 1, but for future climates.
Figures $1 \mathrm{~b}$ and $1 \mathrm{c}$ show the variability ranges of $S P I 12_{\mathrm{p}}$ with some procedures for the present climate projections of MRI-AGCM3.2S and 13 CGCMs in CMIP5. Hereafter, a white cross mark means the SPI average during 25 years, and a white error bar shows the range of $\pm 1 \sigma$ for each run. Since the SPI is based on a standardized normal distribution, the average and standard deviation of the SPI values are zero and one, respectively. Then, five horizontal bars of wide and narrow boxes indicates the 6.68th, 15.87th, 50th, 84.13th and 93.32th percentile of the actual $S P I 12_{\mathrm{p}}$ from lower to higher for each run. These percentile values are the cumulative prob-
ndardized normal distribution, respectively. The both

abilities of $-1.5 \sigma,-1 \sigma$, median, $1 \sigma$, and $1.5 \sigma$ of a standardized normal distribution, respectively. The both

Table 1. Mean annual precipitation averaged in the Angat dam catchment of (a) APHRODITE, (b) MRIAGCM3.2S, 1 present and 4 future climate projections, and (c) CMIP5 models, which have 13 pairs of present (p) and future (f) climate projections. Unit is $\mathrm{mm} /$ year.

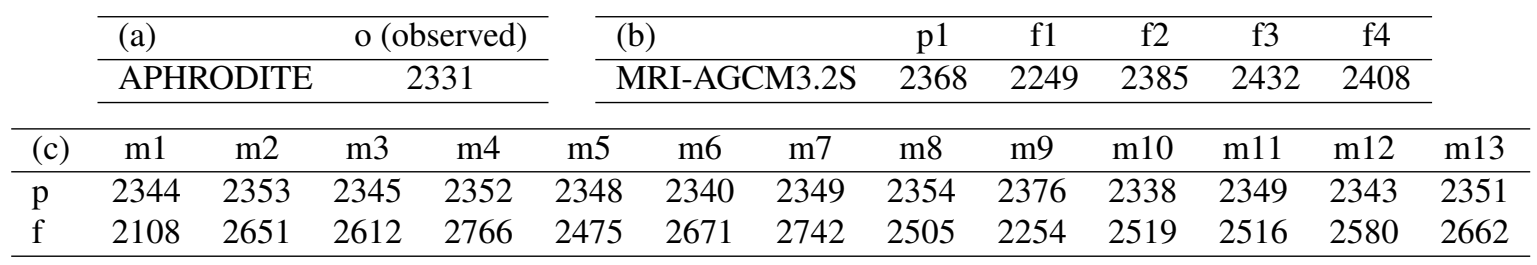


ends of wide box were almost similar to those of error bar, and the median bar was nearby the cross of the average for each run. These small differences within the normal range could be decreased as the number of years in the sample increased. The end of narrow box was not fit to \pm 1.5 of the SPI and asymmetric between dry and wet sides in many runs. This indicates the actual SPI values have a certain skewness.

Figure 2 shows the variability ranges in the future climate projections. They look similar to those in the present climate cases in Figure 1, except for the out of the normal range. Actually, mean annual precipitation in the Angat dam catchment was increased in most of the future climate projections, as shown in Table 1. In spite of the increased mean precipitation, the change of the SPI values looks quite small between the present and future climate projections, as shown in Figures 1 and 2. As result, we conclude that the current SPI approach cannot represent the severity and probability changes of extreme events due to climate change by a simple comparison between $S P I_{\mathrm{p}}$ and $S P I_{\mathrm{f}}$. Therefore, a new approach is needed to evaluate climate change impacts with standardized indices.

\section{SPI LIMITATION IN CLIMATE CHANGE ASSESSMENT}

The limitation of SPI climate change approach is illustrated in Figure 3. In Figure 3, we demonstrate the CDF and SPI values for 12th month at 12-month timescale obtained with MRI-AGCM3.2S runs for present and future climates. Figures $3 \mathrm{a}$ and $3 \mathrm{~b}$ show the CDF and $S P I_{\mathrm{p}}$ of the MRI-AGCM3.2S present climate projection $\mathrm{p} 1$ run, respectively. For the future climate projection, Figures $3 \mathrm{c}$ and $3 \mathrm{~d}$ show the same month and timescale CDF and $S P I_{\mathrm{f}}$. In both Figures $3 \mathrm{~b}$ and $3 \mathrm{~d}$, the moderately and severely dry/wet zones of the SPI are shaded vertically and horizontally with the same colors as in Figures 1 and 2 and are connected with the corresponding cumulative probabilities in Figures $3 \mathrm{a}$ and $3 \mathrm{c}$.

Many climate change SPI studies (e.g., Grillakis et al., 2011; Wang et al., 2011) compare the degrees of severity and probability of drought cases defined by the same SPI threshold between present and future climates. However, the same present and future SPI threshold has different associated precipitation in the CDF s. For example, the present drought defined with $S P I \leq-1$ has annual precipitation less than $2100 \mathrm{~mm}$ in Figure 3a and is less than $1860 \mathrm{~mm}$ for the future climate in Figure 3c. As a result, it is
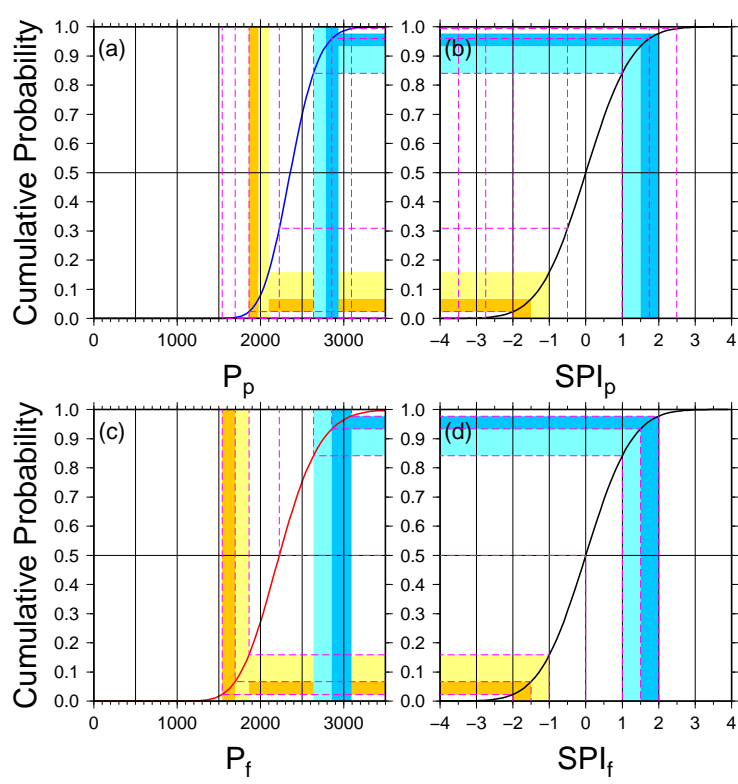

Figure 3. Relationship among (a) CDF of present climate precipitation, (b) SPI of present climate, (c) CDF of future climate precipitation, and (d) SPI of future climate.

more valuable to assess changes of the probability and the degrees of severity in future climate projections on the basis of the corresponding present climate condition. This approach is demonstrated by dashed lines in Figure 3. The dashed lines are connected from $S P I_{\mathrm{f}}$ in Figure $3 \mathrm{~d}$ to $S P I_{\mathrm{p}}$ in Figure $3 \mathrm{~b}$ via the CDFs in Figures $3 \mathrm{c}$ and 3a. From the dashed lines, the annual precipitation of about $2200 \mathrm{~mm}$ is now classified as $S P I_{\mathrm{f}}=0$ (Figures 3c and 3d) and as $S P I_{\mathrm{p}}=-0.5$ (Figures 3a and 3b). Therefore, the drier side of $S P I_{\mathrm{f}} \leq-1$ in the future climate is classified as extremely drought of $S P I_{\mathrm{p}} \leq-2$ on the present climate basis. The moderately and severely wet zones, $1 \leq S P I_{\mathrm{f}}<2$ of the future climate are extended to $1 \leq S P I_{\mathrm{p}}<2.5$ on the present climate basis.

\section{COMPARATIVE SPI APPROACH}

To address this issue, we propose a cSPI approach for the climate change assessment. In our approach, we estimate future climate SPI by substituting precipitation of a future climate as a target dataset for $x$ in the present climate $\mathrm{CDF}$ in equation (1). Both the present and future climate CDFs have the same parameters in equation (1) derived from a present climate condition dataset. The resulted SPI is called a cSPI, $c S P I_{\mathrm{f}, \mathrm{p}}$, where the former and the latter subscripts indicate target and reference datasets, respectively. If the same input datasets are used for both CDFs, the cSPI equals to the traditional SPI, such as $c S P I_{\mathrm{p}, \mathrm{p}}=S P I_{\mathrm{p}}$.

The cSPI approach is illustrated in Figure 4. Figure 4a shows the time series of $c S P I 12_{\mathrm{f}, \mathrm{p}}$ of $\mathrm{f} 1$ run on the 
basis of $\mathrm{p} 1$ run of MRI-AGCM3.2S. The degrees of severity, probability and duration of dry and wet conditions are intuitively understandable in the cSPI values, compared to the SPI values in Figure 2a.

Figures $4 \mathrm{~b}$ and $4 \mathrm{c}$ show the variability ranges of $c S P I 12_{\mathrm{f}, \mathrm{p}}$ of the future climate projections of MRIAGCM3.2S and 13 CGCMs in CMIP5, on the basis of the corresponding present climate projections. As opposed to the traditional SPI in Figures $2 b$ and 2c, the mean and median of each run was shifted from zero, due to the differences of mean precipitation between the present and future climates (Table 1). In Figure 4, a few climate change runs indicate drier average of $c S P I 12_{\mathrm{f}, \mathrm{p}}$ in future climate, while the majority of future projections show wetter future averages. Although the SST ensemble average from f1 to 44 runs of the mean $c S P I 12_{\mathrm{f}, \mathrm{p}}$ was almost zero in MRI-AGCM3.2S, the model ensemble average of that was about 0.55 in CMIP5.

In addition to the mean $c S P I 12_{\mathrm{f}, \mathrm{p}}$, the changes of the variability ranges are important to assess the meteorological drought and flood conditions, as shown by error bars and boxes in Figures $4 \mathrm{~b}$ and $4 \mathrm{c}$. In Figure $4 \mathrm{~b}$, the standard deviations of $c S P I 12_{\mathrm{f}, \mathrm{p}}$ in-
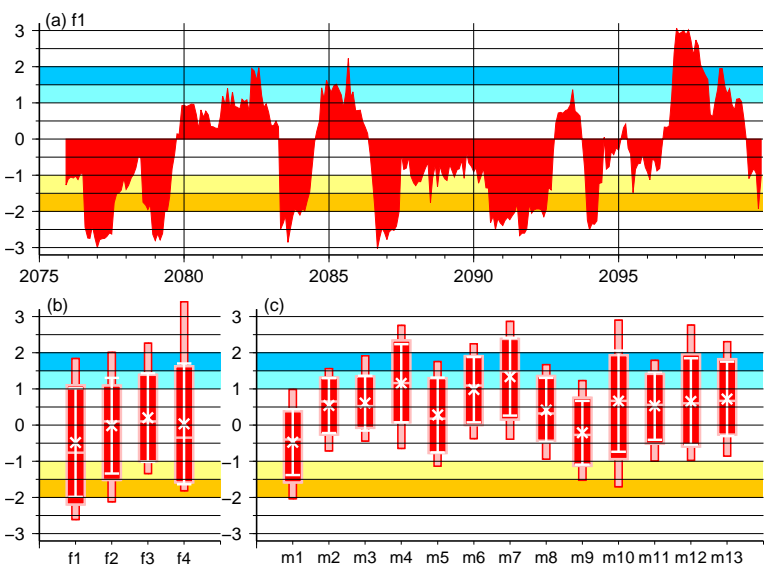

Figure 4. Time series of $c S P I 12_{\mathrm{f}, \mathrm{p}}$ of $\mathrm{f} 1$ run of MRI-AGCM3.2S (a) on the basis of each corresponding present climate run in Figure 1. The variability ranges of $c S P I 12_{\mathrm{f}, \mathrm{p}}$ of future climate runs using (b) MRI-AGCM3.2S and (c) 13 CGCMs in CMIP5. creased in all MRI-AGCM3.2S runs and the probabilities of drier and wetter conditions also increased in MRI-AGCM3.2S RCP8.5 experiments. This is due to the fact that the lower and higher ends of error bars reached to the threshold of -1 and 1, respectively. In Figure $4 \mathrm{c}$, the contributions of the standard deviation change of $c S P I_{\mathrm{f}, \mathrm{p}}$ was smaller compared to MRI-AGCM3.2S runs while the range of $c S P I 12_{\mathrm{f}, \mathrm{p}}$ means is much larger in CMIP5 models. Although the SST ensemble mean of the standard deviation of $c S P I 12_{\mathrm{f}, \mathrm{p}}$ was about 1.4 in MRI-AGCM3.2S, the model ensemble mean of the standard deviation of $c S P I 12_{\mathrm{f}, \mathrm{p}}$ was nearly equal to 1 in CMIP5.

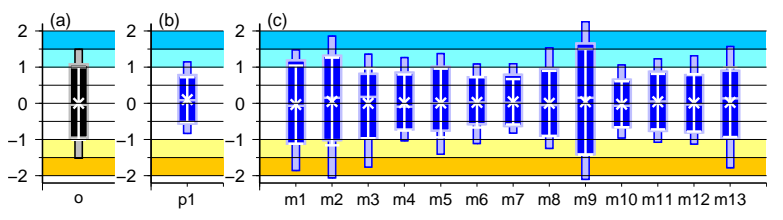

Figure 5. Variability ranges of (a) $S P I 12$ of the observation, and $c S P I 12_{\mathrm{p}, \mathrm{o}}$ of present climate runs using (b) MRI-AGCM3.2S and (c) 13 CGCMs in CMIP5 on the basis of the observation.
Depending on the combination of target and reference datasets, the concept of the cSPI approach is versatile and is useful for many applications besides the comparison between present and future climate projections. For example, we can utilize cSPI to estimate the performance of present climate projections on the basis of the observation dataset, as shown in Figure 5. Compared to $S P I 12_{\text {o }}$ of the observation in Figure 5a, where the subscript "o" means the observation, all of the present climate projections were well bias-corrected in the average base, since the mean $c S P I 12_{\mathrm{p}, \mathrm{o}}$ was nearly equal to zero for each model in Figures $5 \mathrm{~b}$ and $5 \mathrm{c}$. The standard deviation of $c S P I 12_{\mathrm{p}, \mathrm{o}}$ smaller than 1 indicates smaller interannual variability compared to that of the observation, or vice versa. Practically, the mean and standard deviation of $c S P I 12_{\mathrm{p}, \mathrm{o}}$ were quite different from those of the observation before the bias correction (results are not shown). The performance of models and bias correction methods also can be estimated with the cSPI in the same way.

\section{DISCUSSIONS}

In the extension to the cSPI, more extremely dry and wet cases, out of $[-3,3]$ range, were increased since the mean and median of the cSPI were shifted from zero in many cases. However, the accuracy of the SPI value out of $[-3,3]$ was quite low, since the slight difference of cumulative probability in the vicinities of zero and one caused the huge difference of the SPI value, as shown in the CDFs in Figure 3. It was mentioned that the SPI were limited to the range [-3,3] to ensure reasonableness in Stagge et al. (2015).

In the vicinity of zero in cumulative probability, the zero-precipitation issue was more significant in the cSPI than the traditional SPI, because of different conditions between target and reference datasets. The two procedures in Loukas and Vasiliades (2004) were more helpful to avoid the problem partly than the traditional way 
Hasegawa et al., Drought assessment in the Pampanga River basin, the Philippines-Part 2: A comparative SPI...

of the CDF modification in equation (2). Even if the mentioned procedure is used, the case of $c S P I_{\mathrm{f}, \mathrm{p}} \ll-3$ might occur. On the other hand, the problem was more serious in the vicinity of one in cumulative probability. Because of the increased precipitable water due to global warming, the case of $c S P I_{\mathrm{f}, \mathrm{p}} \gg 3$ might occur more frequently in future climate. When there is no solution of such problems, we should analyze the probability change of $c S P I_{\mathrm{f}, \mathrm{p}} \geq 3$ to estimate wet extremes, but it might be hard to assess the severity of the extremes quantitatively. Then, the accuracy problem of extremes might affect the mean and standard deviation of the cSPI. It is more robust to analyze the median and percentile values, if the number of the sample years is large enough. Thus, we displayed not only the mean and standard deviation but also the median and percentile values of the cSPI in this study.

In spite of such limitations, we believe that the cSPI is versatile and valuable to analyze precipitation characteristics in target dataset on the basis of reference dataset, as depending on comparative combinations of target and reference datasets. For example, the cSPI of combination of present climate simulation and observation could estimate the model performance, as mentioned in Section 5. When a certain period in an ongoing longterm observation is defined as a reference, the meteorological drought and flood could be monitored using the cSPI on the fixed basis of the historical reference, without recalculation through the whole period when the newest precipitation is added. For these cases, the cSPI is very useful because the temperature change is not so large within the period treated as present climate.

\section{CONCLUSIONS}

This paper demonstrated a new concept of the comparative SPI (cSPI) to assess the meteorological hazards of droughts and floods under climate change at the Angat dam catchment, the Pampanga River basin, the Philippines. Using the cSPI at 12-month timescale, we estimated the meteorological drought and flood with bias-corrected precipitation dataset in future climate projections under the RCP8.5 emission scenario, on the basis of the corresponding present climate projections of MRI-AGCM3.2S and higher-resolution CGCMs in CMIP5. In SST ensemble runs of MRI-AGCM3.2S, the interannual variability was significantly increased, while the change of the mean of $c S P I 12_{\mathrm{f}, \mathrm{p}}$ depended on the SST distributions. Both meteorological drought and flood probabilities increased in SST ensemble runs under the RCP8.5 condition on the basis of an AMIPtype run of MRI-AGCM3.2S. In the multi-model ensemble in CMIP5, the meteorological drought probability decreased and heavy wet condition increased, since most of the mean and median of $c S P I 12_{\mathrm{f}, \mathrm{p}}$ significantly increased. The cSPI results suggested that the increasing heavy wet condition was more likely in future climate under the RCP8.5 scenario, it would be required to adapt to the heavier wet condition in the Angat dam in the end of the 21 st century. To assess the heavier precipitation in details, the dynamic downscaling of the climate projections is necessary and valuable, while the model uncertainty of GCMs remains in the our meteorological drought assessment.

The cSPI utilizes the same equations of the traditional SPI (McKee et al., 1993), but the cSPI is computed by substituting target datasets in the CDF with parameters derived from a reference dataset. The main advantage of the cSPI is the possibility of evaluating target datasets using the reference dataset features. When a pair of present and future climate projections are applied to reference and target datasets, for example, the meteorological droughts and floods in the future climate are compared on the basis of a present climate projection. Therefore, the cSPI provides us with an alternative point of view when comparing the traditional SPI values between present and future climate projections. In addition, the comparative standardized index concept of the cSPI approach is applicable to the other standardized indices, e.g., the standardized streamflow index (SSI; Vicente-Serrano et al., 2012), the standardized reservoir storage index (SRSI; Gusyev et al., 2015a), and the standardized precipitation evapotranspiration index (SPEI; Vicente-Serrano et al., 2010), which is obtained with various probability distributions. The comparative SRSI could characterize the socio-economic drought more effectively than the traditional indices, and is utilized for the climate change evaluation in Part 3 (Gusyev et al., 2015b) of this study. When using continuous simulations from present to future climates, such as the historical and RCP experiments in CMIP5, the comparative standardized indices could be valuable tools to assess the meteorological, hydrological, and socio-economic droughts in future conditions on the more certain basis of the present climate, by excluding future climate uncertainties from the reference dataset.

\section{ACKNOWLEDGEMENT}

We acknowledge the World Climate Research Programme's Working Group on Coupled Modelling, which is responsible for CMIP, and we thank the climate modeling groups (listed in Section 2 of this paper) for producing and making available their model output. For CMIP the U.S. Department of Energy's Program for 
Hasegawa et al., Drought assessment in the Pampanga River basin, the Philippines-Part 2: A comparative SPI...

Climate Model Diagnosis and Intercomparison provides coordinating support and led development of software infrastructure in partnership with the Global Organization for Earth System Science Portals.

This work was conducted under the framework of the "Precise Impact Assessments on Climate Change" of the Program for Risk Information on Climate Change (SOUSEI Program) supported by the Ministry of Education, Culture, Sports, Science, and Technology-Japan (MEXT).

\section{REFERENCES}

Edwards, D. C. and T. B. McKee (1997). Characteristics of 20th century drought in the united states at multiple time scales. Atmospheric Science Paper No. 634, Climatology Report No. 97-2, Department of Atmospheric Science, Colorado State University.

Grillakis, M. G., A. G. Koutroulis, and I. K. Tsanis (2011). Climate change impact on the hydrology of Spencer Creek watershed in Southern Ontario, Canada. J. Hydrol. 409, 1-19.

Gusyev, M., A. Hasegawa, J. Magome, D. Kuribayashi, H. Sawano, and S. Lee (2015a). Drought assessment in the Pampanga River basin, the Philippines — Part 1: Characterizing a role of dams in historical droughts with standardized indices. In 21st International Congress on Modeling and Simulation, Queensland, Australia.

Gusyev, M., A. Hasegawa, J. Magome, H. Umino, and H. Sawano (2015b). Drought assessment in the Pampanga River basin, the Philippines - Part 3: Evaluating climate change impacts on dam infrastructure with standardized indices. In 21st International Congress on Modeling and Simulation, Queensland, Australia.

Inomata, H., K. Takeuchi, and K. Fukami (2011). Development of a statistical bias correction method for daily precipitation data of GCM20. Annu. J. Hydraul. Engr, JSCE 55, 247-252.

Loukas, A. and L. Vasiliades (2004). Probabilistic analysis of drought spatiotemporal characteristics in Thessaly region, Greece. Natural Hazards and Earth System Sciences 4, 719-731.

McKee, T. B., N. J. Doesken, and J. Kleist (1993). The relationship of drought frequency and duration to timescales. In Proceedings presented at 8th Conference on Applied Climatology, Anaheim, CL, pp. 179184. Am. Met. Soc.

Mizuta, R., O. Arakawa, T. Ose, S. Kusunoki, H. Endo, and A. Kitoh (2014). Classification of CMIP5 future climate responses by the tropical sea surface temperature changes. SOLA 10, 167-171.

Mizuta, R., H. Yoshimura, H. Murakami, M. Matsueda, H. Endo, T. Ose, K. Kamiguchi, M. Hosaka, M. Sugi, S. Yukimoto, S. Kusunoki, and A. Kitoh (2012). Climate simulations using MRI-AGCM3.2 with 20-km grid. J. Meteor. Soc. Japan 90A, 233-258.

Stagge, J. H., L. M. Tallkaksen, L. Gudmundsson, A. F. Van Loon, and K. Stahl (2015). Candidate distributions for climatological drought indices (SPI and SPEI). Int. J. Climatol., W09527.

Taylor, K. E., R. J. Stouffer, and G. A. Meehl (2012). An overview of CMIP5 and the experiment design. Bull. Amer. Meteor. Soc., 485-498.

Vicente-Serrano, S. M., S. Beguería, and J. I. López-Moreno (2010). A multiscalar drought index sensitive to global warming: The standardized precipitation evapotranspiration index. J. Clim. 23, 1696-1718.

Vicente-Serrano, S. M., J. I. López-Moreno, S. Beguería, J. Lorenzo-Lacruz, C. Azorín-Molina, and E. MoránTejeda (2012). Accurate computation of a streamflow drought index. J. Hydrol. Engr. 17(2), 318-332.

Wang, D., M. Hejazi, X. Cai, and A. J. Valocchi (2011). Climate change impact on meteorological, agricultural, and hydrological drought in central Illinois. Water Resour. Res. 47, W09527.

World Meteorological Organization (2012). Standardized precipitation index user guide. WMO-No. 1090. (M. Svoboda, M. Hayes and D. Wood), Geneva.

Yatagai, A., K. Kamiguchi, O. Arakawa, A. Hamada, N. Yasutomi, and A. Kitoh (2012). APHRODITE constructing a long-term daily gridded precipitation dataset for Asia based on a dense network of rain gauges. Bull. Amer. Meteor. Soc., 1401-1415. 